\title{
Novel Two-Dimensional Floating Strip Micromegas Detectors
}

\author{
Felix Klitzner ${ }^{* a}$, Otmar Biebel ${ }^{a}$, Bernhard Flierl ${ }^{a}$, Björn Gillich ${ }^{a}$, Ralf Hertenberger ${ }^{a}$, \\ Philipp Lösel $^{a}$, Johannes Scherzer ${ }^{a}$ and Jonathan Bortfeldt ${ }^{a, b}$ \\ ${ }^{a}$ Ludwig-Maximilians-Universität München, Germany \\ ${ }^{b}$ now at CERN \\ E-mail: Felix.Klitzner@physik.uni-muenchen.de
}

Floating strip Micromegas detectors with one-dimensional readout strips are high-rate capable particle detectors with excellent spatial and temporal resolution, allowing single particle tracking for particle fluxes up to $7 \mathrm{MHz} / \mathrm{cm}^{2}$. A floating strip Micromegas detector collects the amplified ionization charge on copper anode strips being on high voltage, so called floating strips. The charge signal is read out by readout strips, separated by a thin insulating layer from the anode strips. This scheme strongly suppresses the sensitivity of the detector to discharges between the micro-mesh and the floating anode strips, induced by strongly ionizing particles. A novel twodimensional readout has been realized with two layers of readout strips, parallel and perpendicular to the floating strips. We present results from characterizing measurements using a $20 \mathrm{MeV}$ proton beam. Charge signals were recorded with APV25 front-end boards, allowing for single strip readout with pulse height and timing information. To investigate hardware multiplexing schemes, advantageous for large area detector systems, we have interconnected groups of readout strips to single electronics channels, studying the signal pulse height. Additionally inclined detectors allowed for testing $\mu$ TPC reconstruction in both readout layers. We also report on alternative designs of the two-dimensional floating strip anode, where readout strip widths and geometry of both readout layers within the PCB have been varied, and its tests with $5.9 \mathrm{keV}$ X-rays emitted by a ${ }^{55} \mathrm{Fe}$ source as well as with $22 \mathrm{MeV}$ protons.

38th International Conference on High Energy Physics

3-10 August 2016

Chicago, USA

${ }^{*}$ Speaker. 


\section{Introduction}

Floating strip Micromegas [1] [2] are high-rate capable gaseous particle detectors with excellent spatial and temporal resolution, allowing single particle tracking for particle fluxes up to $7 \mathrm{MHz} / \mathrm{cm}^{2}$ [2]. The copper anode strips are individually connected to high-voltage via high-ohmic resistors. Ionization charge is collected in the typically $6 \mathrm{~mm}$ wide drift region between cathode and mesh. After gas amplification, the signal couples to readout strips (see fig. 1), insulated by a thin layer of Kapton. This scheme of 'floating' strips strongly suppresses the sensitivity of the detector to discharges between mesh and anode, induced by e.g. strongly ionizing particles. One-dimensional floating strip Micromegas have been successfully tested in various beam environments, showing optimum spatial and temporal resolution of $49 \mu \mathrm{m}$ at a strip pitch of $0.25 \mathrm{~mm}$ [2] and $8 \mathrm{~ns}$, respectively. With low material budget and an anode structure as thin as $0.01 \cdot X_{0}$, multiple scattering is minimized, thus making the detector attractive to medical imaging applications [3]. In the following sections, two-dimensional floating strip Micromegas are introduced. Three possible anode designs are presented and detector responses in both dimensions with respect to strip layer pulse height and angle reconstruction capability are shown.

\section{Two-Dimensional Floating Strip Micromegas}

Three different two-dimensional floating strip Micromegas anode layouts have been tested. The floating strips feature a strip pitch of $0.5 \mathrm{~mm}$ and a width of $0.3 \mathrm{~mm}$. Readout strip width and location have been varied within the three designs. Fig. 2 illustrates anode layout 1 and anode layout 3.
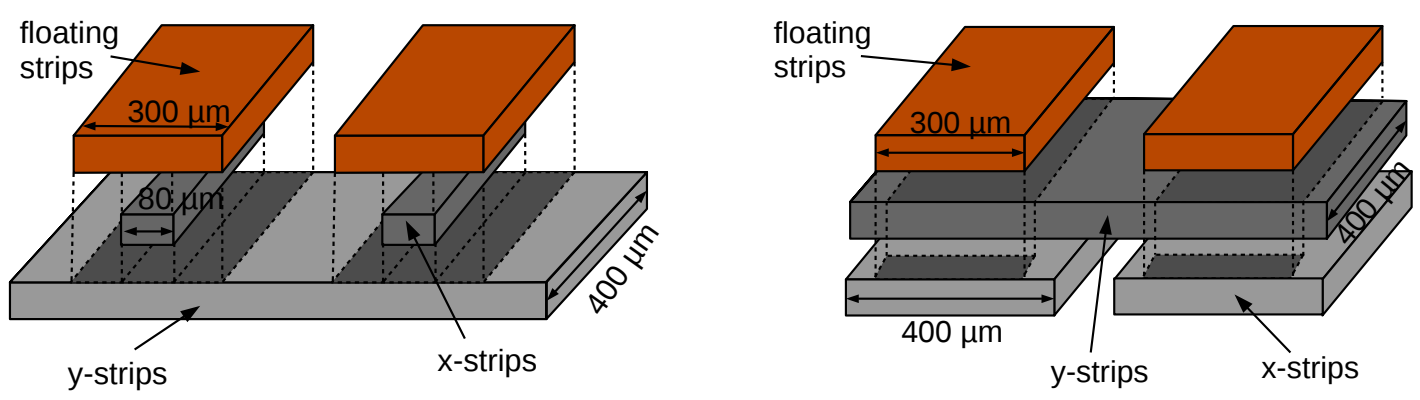

Figure 2: Two out of the three tested two-dimensional floating strip Micromegas anode layouts. Left figure: anode layout 1 , right figure: anode layout 3 .

Anode layout 2 has the same strip orientation as layout 1 but a $x$-strip width of $300 \mu \mathrm{m}$ instead of $80 \mu \mathrm{m}$. While the active area of anode layout 1 covers $64 \times 64 \mathrm{~mm}^{2}$ with 128 strips, anode layout 2 and 3 cover an area of $192 \times 192 \mathrm{~mm}^{2}$ with 384 strips. The three $35 \mu \mathrm{m}$ high strip layers on the anode PCB are insulated by two layers of $25 \mu \mathrm{m}$ Kapton, resulting in a $0.15 \mathrm{~mm}$ thin anode. Data has been recorded with the Scalable Readout System [4] interfacing APV25 front-end boards, 
allowing for single strip read out with pulse height and timing information. The responses of typical signals on anode layout 1 and 3 from $20 \mathrm{MeV}$ protons are shown in fig. 3 . The signals on both anode
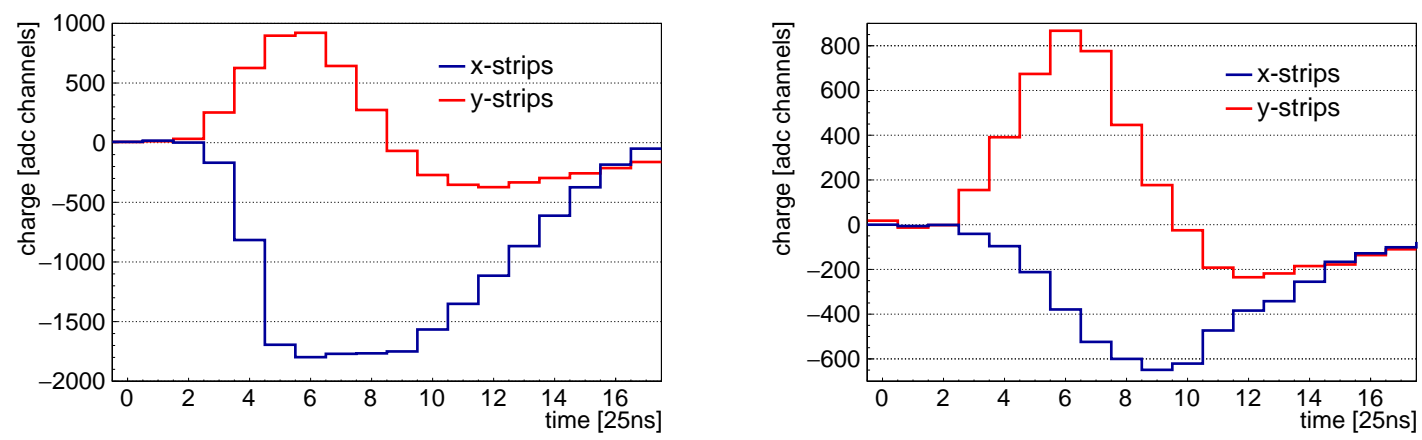

Figure 3: Signal on the strip with maximum charge as a function of time. Left figure: Signal on anode layout 1 at $E_{a m p}=35.3 \mathrm{kV} / \mathrm{cm}$ and $E_{\text {drift }}=0.33 \mathrm{kV} / \mathrm{cm}(20 \mathrm{MeV}$ p). Right figure: Signal on anode layout 3 at $\mathrm{E}_{\mathrm{amp}}=36.7 \mathrm{kV} / \mathrm{cm}$ and $\mathrm{E}_{\mathrm{drift}}=0.33 \mathrm{kV} / \mathrm{cm}(22 \mathrm{MeV} \mathrm{p})$.

layouts show opposite signal polarity on $x$ - and $y$-strips as well as a shorter pulse duration on the $y$-strips. On the $x$-strips of anode layout 1 a signal saturation at about 1800 adc channels is visible due to the limited dynamic range of the APV25. Whereas the pulse height on the $x$-strips for anode layout 1 is considerably higher than on the $y$-strips, the $y$-strip signal is higher than the $x$-strip signal for anode layout 3 .

\section{Pulse Height Behaviour}

In the following, the pulse height ratio $\mathrm{ph}_{\mathrm{y}} / \mathrm{ph}_{\mathrm{x}}$ is being investigated as a function of the amplification field and the anode layout. The pulse height $\mathrm{ph}=\sum_{\text {cluster }} q_{\text {strip }}$ is the summed charge of the signal, where $\mathrm{q}_{\text {strip }}$ is the measured charge on each strip in the cluster. Fig. 4 shows the pulse height ratio for all three anode layouts as a function on the amplification field. While layout 1 and 3 have been characterized with $20 \mathrm{MeV}$ protons at

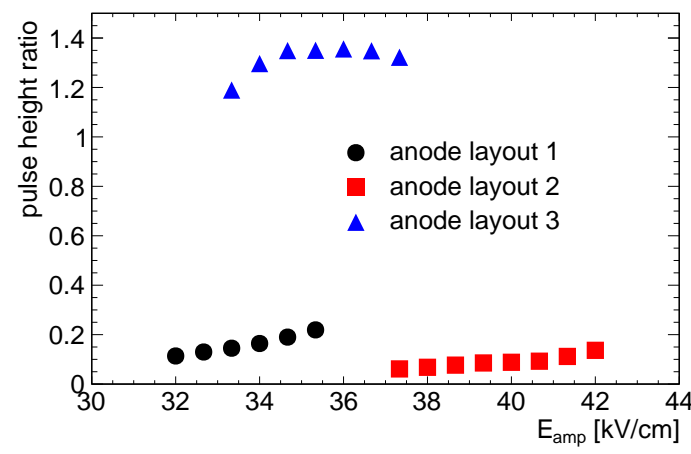

Figure 4: Pulse height ratio $\mathrm{ph}_{\mathrm{y}} / \mathrm{ph}_{\mathrm{x}}$ as a function of the amplification field for the three anode layouts.

the Tandem accelerator in Garching, anode layout 2 was characterized using a ${ }^{55} \mathrm{Fe}$-source. It can be seen that only for layout 3 the signal pulse height on both strip layers is of the same order and converges to a factor of $\mathrm{ph}_{\mathrm{y}} / \mathrm{ph}_{\mathrm{x}} \approx 1.3$ for $\mathrm{E}_{\mathrm{amp}} \geq 34 \mathrm{kV} / \mathrm{cm}$. For anode layouts 1 and 2 , the $x$-layer signals were always in saturation for all tested amplification fields. As this is typically unwanted due to degrading the spatial resolution in this dimension, anode layout 3 shows the best signal reconstruction capabilities in both dimensions simultaneously.

\section{Simultaneous Angle Reconstruction in Both Dimensions}

To test the angle reconstruction of inclined particle tracks, the detector has been tilted with respect to a $22 \mathrm{MeV}$ proton beam, see fig. 5. For the angle reconstruction a so-called $\mu$ TPC method has been used, inspired by the analysis of Time-Projection-Chambers. Anode layout 3 shows an 
optimum angular resolution for the $x$-layer of $\left(\begin{array}{c}+2.9 \\ -2.1\end{array}\right)^{\circ}$ and for the $y$-layer of $\left(\begin{array}{c}+2.2 \\ -1.8\end{array}\right)^{\circ}$ for small drift field configurations.
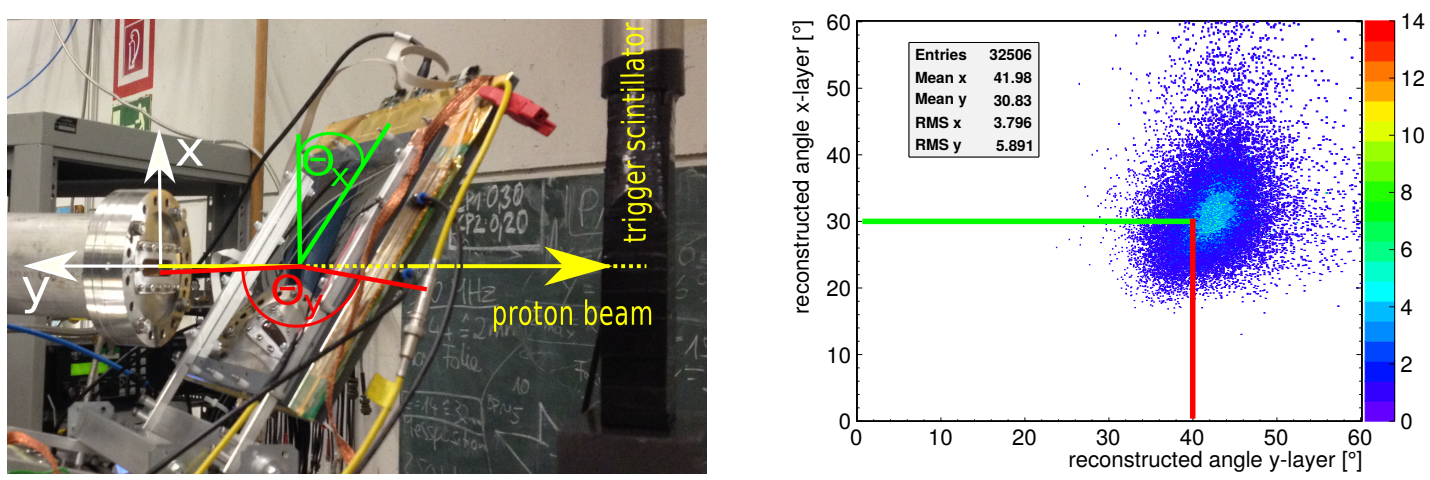

Figure 5: Left figure: Photograph of the setup at the Tandem accelerator in Garching with a $22 \mathrm{MeV}$ proton beam. The detector has been tilted $\Theta_{\mathrm{x}}=(30 \pm 1)^{\circ}$ with respect to the $x$-layer and $\Theta_{\mathrm{y}}=(40 \pm 2)^{\circ}$ with respect to the $y$-layer. Right figure: Reconstructed angle in both readout layers with anode layout 3 at $\mathrm{E}_{\mathrm{amp}}=38.3 \mathrm{kV} / \mathrm{cm}$ and $\mathrm{E}_{\mathrm{drift}}=0.08 \mathrm{kV} / \mathrm{cm}$.

Anode layout 1 shows slightly better angle reconstruction capabilities due to a saturated signal on the $x$-layer and a higher signal on the $y$-layer. Thus the $x$-layer shows an optimum angular resolution of $\left(\begin{array}{c}+1.6 \\ -1.2\end{array}\right)^{\circ}$ and the $y$-layer of $\left(\begin{array}{c}+1.5 \\ -1.2\end{array}\right)^{\circ}$, again for small drift field configurations.

\section{Summary}

We have presented three different two-dimensional floating strip Micromegas anode layouts. Signals on both readout strip layers show opposite signal polarity and a shorter duration on the $y$ layer. Studies on the pulse height ratio for both readout layers showed best results for anode layout 3 with $\mathrm{ph}_{\mathrm{y}} / \mathrm{ph}_{\mathrm{x}} \approx 1.3$ for $\mathrm{E}_{\mathrm{amp}} \geq 34 \mathrm{kV} / \mathrm{cm}$. It could be shown that the reconstruction of inclined particle tracks works independently for both readout strip layers with anode layout 1 and 3 .

\section{Acknowledgments}

We acknowledge the support by the DFG Excellence Cluster 'Origin and Structure of the Universe' and want to thank the staff of the MLL Tandem accelerator.

\section{References}

[1] Y. Giomataris et al., Micromegas: a high-granularity position sensitive gaseous detector for high particle-flux environments, Nucl. Instr. Meth. A 37629 - 35 (1996).

[2] J. Bortfeldt, The Floating Strip Micromegas Detector: Versatile Particle Detectors for High-rate Applications (Springer Theses), Springer, ISBN 978-3-319-18892-8, 2015.

[3] J. Bortfeldt et al., Low material budget floating strip Micromegas for ion transmission radiography, Nucl. Instr. Meth. A, ISSN 0168-9002, http://dx.doi.org/10.1016/j.nima.2016.05.003, 2016.

[4] S. Martoiu and H. Muller and A. Tarazona and J. Toledo, Developement of the scalable readout system for micro-pattern gas detectors and other applications, JINST, 8 (03):C03015, 2013. 